\title{
Several conservative compact schemes for a class of nonlinear Schrödinger equations with wave operator
}

\author{
Xiujun Cheng ${ }^{1,2}$ and Fengyan $\mathrm{Wu}^{1,2^{*}}$
}

\section{"Correspondence:}

fywuhust@163.com

'School of Mathematics and

Statistics, Huazhong University of

Science and Technology, Wuhan,

China

${ }^{2}$ Center for Mathematical Sciences, Huazhong University of Science and

Technology, Wuhan, China

\begin{abstract}
In this paper, several different conserving compact finite difference schemes are developed for solving a class of nonlinear Schrödinger equations with wave operator. It is proved that the numerical solutions are bounded and the numerical methods can achieve a convergence rate of $\mathcal{O}\left(\tau^{2}+h^{4}\right)$ in the maximum norm. Moreover, by applying Richardson extrapolation, the proposed methods have a convergence rate of $\mathcal{O}\left(\tau^{4}+h^{4}\right)$ in the maximum norm. Finally, several numerical experiments are presented to illustrate the theoretical results.
\end{abstract}

MSC: $65 \mathrm{~N} 06 ; 65 \mathrm{~N} 12$

Keywords: Nonlinear Schrödinger equations with wave operator; Discrete conservation laws; Stability; Convergence

\section{Introduction}

This paper is concerned with the construction of several conservative compact schemes for the following generalized nonlinear Schrödinger equation (NLSE) with wave operator:

$$
\begin{array}{ll}
u_{t t}-u_{x x}+\mathrm{i} \alpha u_{t}+\beta(x) f\left(|u|^{2}\right) u=0, & x \in(0, L), t \in(0, T), \\
u(x, 0)=\phi_{0}(x), \quad u_{t}(x, 0)=\phi_{1}(x), & x \in[0, L], \\
u(0, t)=u(L, t)=0, \quad t \in[0, T], &
\end{array}
$$

where $u(x, t)$ is a complex function, $\alpha$ is a real constant, $\beta$ and $f$ are given real functions, and $\mathrm{i}^{2}=-1$. NLSE is one of the most important mathematical models with many applications in different fields such as plasma physics [1], nonlinear optics [2-5], and bimolecular dynamics [6-8]. One remarkable feature of the model is its conservation law having the form of

$$
E(t)=\left\|u_{t}\right\|_{L_{2}}^{2}+\left\|u_{x}\right\|_{L_{2}}^{2}+\int_{0}^{L} \beta(x) F\left(|u|^{2}\right) d x=E(0)
$$

where $F(s)=\int_{0}^{s} f(z) d z$.

(c) The Author(s) 2018. This article is distributed under the terms of the Creative Commons Attribution 4.0 International License (http://creativecommons.org/licenses/by/4.0/), which permits unrestricted use, distribution, and reproduction in any medium, provided you give appropriate credit to the original author(s) and the source, provide a link to the Creative Commons license, and indicate if changes were made. 
In the past several years, much attention has been paid to developing effective numerical methods to solve the NLSE. For example, Bao and Cai [9] established uniform error estimates of finite difference methods for NLSEs with wave operator. Sun and Wang [10] investigated linearized finite difference methods for solving the NLSE. Chang et al. [11] presented several linearized finite difference schemes by applying an extrapolation technique to the real coefficient of the nonlinear term. Goubet and Hamraoui [12] presented both numerical and theoretical invariability of energy and mass with finite time for twodimensional cubic nonlinear Schrödinger equations with a radial defect. Generally speaking, the computational cost can be reduced by applying the linearized numerical methods. As a result, the linearized methods have been extensively investigated for many different nonlinear differential equations, e.g., [13-21]. However, the nonconservative linearized schemes may give blow-up solutions [22].

Recently, many numerical methods have been developed based on the conservation laws of (1.1). For example, Brugnano et al. [23] considered a class of energy-conserving Hamiltonian boundary value methods for the equations. In [24], Zhang and Chang proposed a four-level explicit and conservative scheme. Wang and Zhang [25] developed some different conservative schemes based on some special techniques on the nonlinear terms. $\mathrm{Hu}$ and Chan [26] further considered a conservative difference scheme for two-dimensional NLSE. In [24, 26], the proposed methods have second order accuracy in spatial direction. In order to improve accuracy in spatial direction, Guo et al. [27] and Cao et al. [28] introduced the energy conserving LDG methods and obtained optimal convergence or superconvergence of the method. Li et al. [29,30] introduced the compact finite difference methods and investigated fully discrete numerical schemes for cubic NLSE with wave operator (i.e., $f(s)=s$ ). As far as we know, there are few results on construction of conservative compact finite difference methods for the generalized NLSE with wave operator (1.1).

In this study, several compact finite difference schemes are developed for solving the generalized NLSE with wave operator (1.1). It is shown that the fully discrete numerical methods conserve the discrete energy. Then, the boundedness of numerical solutions and the stability of numerical methods are obtained. It is also proved that the numerical methods can attain a convergence rate of $\mathcal{O}\left(\tau^{2}+h^{4}\right)$ in the maximum norm. Here and below, $\tau$ and $h$ are respectively the temporal and spatial stepsizes. Besides, by applying the Richardson extrapolation algorithm, the proposed methods have a convergence rate of $\mathcal{O}\left(\tau^{4}+h^{4}\right)$ in the maximum norm. Finally, several numerical experiments are proposed to illustrate all the theoretical results.

The rest of the paper is organized as follows. In Sect. 2, a compact difference scheme is given. In Sect. 3, the discrete conservation law of the compact scheme is obtained. In Sect. 4 , the boundedness of numerical solutions is obtained. In Sect. 5, stability and convergence are proved. In Sect. 6, several conservative compact schemes are constructed for the nonlinear Schrödinger equation with wave operator. Moreover, the Richardson extrapolation technique is used. In the last section, numerical experiments are presented to support theoretical results.

Throughout the paper, we set $C$ as a general positive constant independent of mesh sizes, which may be changed under different circumstances. 


\section{Finite difference scheme}

Let $\tau=\frac{T}{N}$ and $h=\frac{L}{J}$ be the temporal and spatial stepsizes, respectively, where $J$ and $N$ are given positive integers. Denote $t_{n}=n \tau(0 \leq n \leq N), x_{j}=j h(0 \leq j \leq J), \Omega_{\tau}=\left\{t_{n} \mid 0 \leq n \leq N\right\}$, and $\Omega_{h}=\left\{x_{j} \mid 0 \leq j \leq J\right\}$. Let $\mathcal{W}_{h}^{0}=\left\{w_{j}^{n} \mid w_{0}^{n}=w_{J}^{n}=0, j=1,2, \ldots, J-1, n=1, \ldots, N-1\right\}$ be a grid function space defined on $\Omega_{h} \times \Omega_{\tau}$. Define

$$
\begin{aligned}
& \delta_{x} w_{j}^{n}=\frac{w_{j+1}^{n}-w_{j}^{n}}{h}, \quad \delta_{\bar{x}} w_{j}^{n}=\frac{w_{j}^{n}-w_{j-1}^{n}}{h}, \\
& \delta_{x}^{2} w_{j}^{n}=\delta_{x} \delta_{\bar{x}} w_{j}^{n}=\frac{1}{h^{2}}\left(w_{j+1}^{n}-2 w_{j}^{n}+w_{j-1}^{n}\right), \\
& \delta_{\hat{t}} w_{j}^{n}=\frac{w_{j}^{n+1}-w_{j}^{n-1}}{2 \tau}, \quad \delta_{\bar{t}} w_{j}^{n}=\frac{w_{j}^{n}-w_{j}^{n-1}}{\tau}, \\
& \delta_{t} w_{j}^{n}=\frac{w_{j}^{n+1}-w_{j}^{n}}{\tau}, \\
& \delta_{t}^{2} w_{j}^{n}=\delta_{t} \delta_{\bar{t}} w_{j}^{n}=\frac{1}{\tau^{2}}\left(w_{j}^{n+1}-2 w_{j}^{n}+w_{j}^{n-1}\right), \\
& A_{h} w_{j}^{n}=\frac{1}{12}\left(w_{j-1}^{n}+10 w_{j}^{n}+w_{j+1}^{n}\right), \\
& \left(\mathbf{w}^{n}, \mathbf{v}^{n}\right)=h \sum_{j=1}^{J-1} w_{j}^{n} \bar{v}_{j}^{n}, \quad\left\|\mathbf{w}^{n}\right\|=\sqrt{h \sum_{j=1}^{J-1}\left|w_{j}^{n}\right|^{2},} \\
& \left\|\delta_{x} \mathbf{w}^{n}\right\|=\sqrt{h \sum_{j=0}^{J-1}\left|\delta_{x} w_{j}^{n}\right|^{2}}, \quad\left\|\mathbf{w}^{n}\right\|_{\infty}=\max _{1 \leq j \leq J-1}\left|w_{j}^{n}\right|,
\end{aligned}
$$

where $\mathbf{w}^{n}=\left(w_{1}^{n}, \ldots, w_{J-1}^{n}\right)^{T}, \mathbf{v}^{n}=\left(v_{1}^{n}, \ldots, v_{J-1}^{n}\right)^{T}$.

At the grid point $\left(x_{j}, t_{n}\right)$, we define $U_{j}^{n}$ as the exact solution and $u_{j}^{n}$ as the numerical solution. We also assume that the exact solution of problem (1.1)-(1.3) satisfies

$$
\max \left\{\left\|\mathbf{U}^{n}\right\|,\left\|\delta_{x} \mathbf{U}^{n}\right\|,\left\|\mathbf{U}^{n}\right\|_{\infty}\right\} \leq C
$$

Now, we present a compact difference scheme for problem (1.1)-(1.3) as follows:

$$
\begin{aligned}
& A_{h} \delta_{t}^{2} u_{j}^{n}-\delta_{x}^{2} u_{j}^{n}+\mathrm{i} \alpha A_{h} \delta_{\hat{t}} u_{j}^{n}+A_{h}\left[\beta\left(x_{j}\right) \frac{F\left(\left|u_{j}^{n+1}\right|^{2}\right)-F\left(\left|u_{j}^{n-1}\right|^{2}\right)}{\left|u_{j}^{n+1}\right|^{2}-\left|u_{j}^{n-1}\right|^{2}} \frac{u_{j}^{n+1}+u_{j}^{n-1}}{2}\right]=0, \\
& \quad 1 \leq j \leq J-1,1 \leq n \leq N-1, \\
& u_{j}^{0}=\phi_{0}\left(x_{j}\right), \quad \delta_{\hat{t}} u\left(x_{j}, 0\right)=\phi_{1}\left(x_{j}\right), \quad 0 \leq j \leq J, \\
& u_{0}^{n}=u_{J}^{n}=0, \quad 0 \leq n \leq N .
\end{aligned}
$$

Denote

$$
\begin{aligned}
& \mathbf{u}^{n}=\left(u_{1}^{n}, \ldots, u_{J-1}^{n}\right)^{T}, \\
& G\left(\left|\mathbf{u}^{n}\right|^{2}\right)=\operatorname{diag}\left(\beta\left(x_{1}\right) \frac{F\left(\left|u_{1}^{n+1}\right|^{2}\right)-F\left(\left|u_{1}^{n-1}\right|^{2}\right)}{\left|u_{1}^{n+1}\right|^{2}-\left|u_{1}^{n-1}\right|^{2}}, \ldots, \beta\left(x_{J-1}\right) \frac{F\left(\left|u_{J-1}^{n+1}\right|^{2}\right)-F\left(\left|u_{J-1}^{n-1}\right|^{2}\right)}{\left|u_{J-1}^{n+1}\right|^{2}-\left|u_{J-1}^{n-1}\right|^{2}}\right)^{T},
\end{aligned}
$$




$$
\begin{aligned}
\mathbf{S} & =\left(\begin{array}{cccccc}
0 & 1 & 0 & \ldots & 0 & 0 \\
1 & 0 & 1 & \ldots & 0 & 0 \\
\vdots & \vdots & \vdots & & \vdots & \vdots \\
0 & 0 & 0 & \ldots & 0 & 1 \\
0 & 0 & 0 & \ldots & 1 & 0
\end{array}\right)_{(J-1) \times(J-1)} \\
\mathbf{M} & =\frac{1}{12}(10 \mathbf{I}+\mathbf{S}), \quad \mathbf{A}=\frac{1}{h^{2}}(-2 \mathbf{I}+\mathbf{S}),
\end{aligned}
$$

where I is an identity matrix.

Since $\mathbf{M}$ is a symmetric positive definite matrix, there exists a real symmetric positive definite matrix $\mathbf{H}$ such that $\mathbf{H}=\mathbf{M}^{-1}$. Then scheme (2.2)-(2.4) can be written in the following vector form:

$$
\begin{aligned}
& \delta_{t}^{2} \mathbf{u}^{n}-\mathbf{H A} \mathbf{u}^{n}+\mathrm{i} \alpha \delta_{\hat{t}} \mathbf{u}^{n}+G\left(\left|\mathbf{u}^{n}\right|^{2}\right) \frac{\mathbf{u}^{n+1}+\mathbf{u}^{n-1}}{2}=0, \quad 1 \leq n \leq N-1, \\
& u_{j}^{0}=\phi_{0}\left(x_{j}\right), \quad \delta_{\hat{t}} u_{j}^{0}=\phi_{1}\left(x_{j}\right), \quad 0 \leq j \leq J, \\
& u_{0}^{n}=u_{J}^{n}=0, \quad 0 \leq n \leq N .
\end{aligned}
$$

\section{Discrete conservation law}

In this section, we will show that the numerical scheme owns the discrete conservation law. First of all, we introduce some lemmas, which will assist a lot in the proof of the main result.

Lemma 3.1 (cf. [31]) The eigenvalues $\lambda_{S}^{j}$ of matrix $\mathbf{S}$ are $2 \cos \left(\frac{j \pi}{J}\right)(j=1,2, \ldots, J-1)$. Then the eigenvalues of matrices $\mathbf{H}, \mathbf{A}$, and $\mathbf{H A}$ are $\frac{12}{10+\lambda_{S}^{j}}, \frac{-2+\lambda_{S}^{j}}{h^{2}}, \frac{12\left(-2+\lambda_{S}^{j}\right)}{h^{2}\left(10+\lambda_{S}^{j}\right)}(j=1,2, \ldots, J-1)$, respectively.

Lemma 3.2 For any mesh function $\mathbf{u} \in \mathcal{W}_{h}^{0}$ and real symmetric positive definite matrices $\mathbf{H}$ and $\mathbf{A}$, we obtain that $-\mathbf{H A}$ is a symmetric positive definite matrix and

$$
-(\mathbf{H A u}, \mathbf{u})=\|\mathbf{R} \mathbf{u}\|^{2},
$$

where $\mathbf{R}$ is obtained by Cholesky decomposition for $-\mathbf{H A}$, denoted as $\mathbf{R}=\operatorname{Chol}(-\mathbf{H A})$.

Proof It follows from $\mathbf{M}=\frac{1}{12}(10 \mathbf{I}+\mathbf{S})$ and $\mathbf{A}=\frac{1}{h^{2}}(-2 \mathbf{I}+\mathbf{S})$ that

$$
\mathbf{M A}=\frac{1}{12 h^{2}}(10 \mathbf{I}+\mathbf{S})(-2 \mathbf{I}+\mathbf{S})=\frac{1}{12 h^{2}}\left(-20 \mathbf{I}+8 \mathbf{S}+\mathbf{S}^{2}\right)=\mathbf{A M}
$$

Therefore, $\mathbf{A H}=\mathbf{H A}$, which implies that $(-\mathbf{A H})^{T}=-\mathbf{A H}$.

In virtue of Lemma 3.1, we can obtain

$$
0 \leq \lambda_{-H A}^{j}=-\frac{12\left(-2+\lambda_{S}^{j}\right)}{h^{2}\left(10+\lambda_{S}^{j}\right)} \leq \frac{6}{h^{2}} \quad(j=1,2, \ldots, J-1) .
$$

Hence, $-\mathbf{H A}$ is a symmetric positive definite matrix. The proof is completed. 
Define

$$
\begin{aligned}
E^{n}= & \left\|\delta_{t} \mathbf{u}^{n}\right\|^{2}+\frac{1}{2}\left(\left\|\mathbf{R} \mathbf{u}^{n}\right\|^{2}+\left\|\mathbf{R} \mathbf{u}^{n+1}\right\|^{2}\right)-\frac{\tau^{2}}{2}\left\|\mathbf{R} \delta_{t} \mathbf{u}^{n}\right\|^{2} \\
& +\frac{h}{2} \sum_{j=1}^{J-1} \beta_{j}\left[F\left(\left|u_{j}^{n}\right|^{2}\right)+F\left(\left|u_{j}^{n+1}\right|^{2}\right)\right] .
\end{aligned}
$$

Then we get the following energy preserving property for the fully discrete numerical scheme (2.7)-(2.9).

Theorem 3.3 The numerical solutions obtained by the fully discrete numerical scheme (2.7)-(2.9) admit: for all $n \geq 0, E^{n}=E^{0}$.

Proof Taking the inner product on both sides of (2.7) with $\mathbf{u}^{n+1}-\mathbf{u}^{n-1}$ and considering the real part, we arrive at

$$
\begin{aligned}
& \operatorname{Re}\left(\delta_{t}^{2} \mathbf{u}^{n}, \mathbf{u}^{n+1}-\mathbf{u}^{n-1}\right)=\left\|\delta_{t} \mathbf{u}^{n}\right\|^{2}-\left\|\delta_{t} \mathbf{u}^{n-1}\right\|^{2}, \\
& \operatorname{Re}\left(\mathbf{H A} \mathbf{u}^{n}, \mathbf{u}^{n+1}-\mathbf{u}^{n-1}\right) \\
& \quad=-\frac{1}{2}\left(\left\|\mathbf{R} \mathbf{u}^{n+1}\right\|^{2}-\left\|\mathbf{R} \mathbf{u}^{n-1}\right\|^{2}\right)+\frac{\tau^{2}}{2}\left(\left\|\mathbf{R} \delta_{t} \mathbf{u}^{n}\right\|^{2}-\left\|\mathbf{R} \delta_{t} \mathbf{u}^{n-1}\right\|^{2}\right), \\
& \operatorname{Re}\left(\mathrm{i} \alpha \delta_{t} \mathbf{u}^{n}, \mathbf{u}^{n+1}-\mathbf{u}^{n-1}\right)=0, \\
& \operatorname{Re}\left(G\left(\left|\mathbf{u}^{n}\right|^{2}\right) \frac{\mathbf{u}^{n+1}+\mathbf{u}^{n-1}}{2}, \mathbf{u}^{n+1}-\mathbf{u}^{n-1}\right) \\
& \quad=\frac{h}{2} \sum_{j=1}^{J-1} \beta_{j}\left[F\left(\left|u_{j}^{n+1}\right|^{2}\right)-F\left(\left|u_{j}^{n-1}\right|^{2}\right)\right] .
\end{aligned}
$$

From (3.3)-(3.6), we have

$$
\begin{gathered}
\left\|\delta_{t} \mathbf{u}^{n}\right\|^{2}-\left\|\delta_{t} \mathbf{u}^{n-1}\right\|^{2}+\frac{1}{2}\left(\left\|\mathbf{R} \mathbf{u}^{n+1}\right\|^{2}-\left\|\mathbf{R} \mathbf{u}^{n-1}\right\|^{2}\right) \\
-\frac{\tau^{2}}{2}\left(\left\|\mathbf{R} \delta_{t} \mathbf{u}^{n}\right\|^{2}-\left\|\mathbf{R} \delta_{t} \mathbf{u}^{n-1}\right\|^{2}\right) \\
+\frac{h}{2} \sum_{j=1}^{J-1} \beta_{j}\left[F\left(\left|u_{j}^{n+1}\right|^{2}\right)-F\left(\left|u_{j}^{n-1}\right|^{2}\right)\right]=0,
\end{gathered}
$$

which further implies that, for $n \geq 1$,

$$
E^{n}=E^{n-1}
$$

Therefore, the conclusion holds.

\section{Boundedness of numerical solutions}

In this section, we present the boundedness of numerical solutions. 
Lemma 4.1 (see [32]) For any mesh function $\mathbf{u}, \mathbf{v} \in \mathcal{W}_{h}^{0}$, there is the identity

$$
-h \sum_{j=1}^{J-1}\left(\delta_{x}^{2} u_{j}\right) \bar{v}_{j}=h \sum_{j=0}^{J-1}\left(\delta_{x} u_{j}\right)\left(\delta_{x} \bar{v}_{j}\right)
$$

which implies that $-(\mathbf{A u}, \mathbf{u})=\left\|\delta_{x} \mathbf{u}\right\|^{2}$.

Lemma 4.2 (cf. [33]) For any symmetric matrix $\mathbf{N}$, the property of Rayleigh-Ritz ratio is

$$
\min \left[\lambda_{N}\right] \leq \frac{(\mathbf{N x}, \mathbf{x})}{(\mathbf{x}, \mathbf{x})} \leq \max \left[\lambda_{N}\right]
$$

where $(\mathbf{x}, \mathbf{y})$ indicates the inner product of $\mathbf{x}$ and $\mathbf{y}, \min \left[\lambda_{N}\right]$ and $\max \left[\lambda_{N}\right]$ denote the smallest and largest eigenvalue of matrix $\mathbf{N}$, respectively.

Lemma 4.3 For any mesh function $\mathbf{u} \in \mathcal{W}_{h}^{0}$, it holds that $-(\mathbf{A u}, \mathbf{u}) \leq-(\mathbf{H A u}, \mathbf{u})$. That is, $\left\|\delta_{x} \mathbf{u}\right\| \leq\|\mathbf{R u}\|$.

Proof It follows from $\mathbf{H A}=\mathbf{A H}$ and $\mathbf{A}^{T}=\mathbf{A}$ that $\mathbf{A}-\mathbf{A H}$ is a symmetric matrix. According to $\mathbf{S} x=\lambda_{S}^{j} x$, we obtain

$$
\mathbf{H} x=\frac{12}{10+\lambda_{S}^{j}} x, \quad \mathbf{A} x=\frac{1}{h^{2}}\left(-2+\lambda_{S}^{j}\right) x .
$$

Therefore,

$$
(\mathbf{I}-\mathbf{H}) x=\left(1-\frac{12}{10+\lambda_{j}^{s}}\right) x=\frac{1}{h^{2}}\left(1-\frac{12}{10+\lambda_{S}^{j}}\right)\left(-2+\lambda_{S}^{j}\right) \mathbf{A}^{-1} x .
$$

Then the eigenvalues of $\mathbf{A}-\mathbf{A H}$ are given by $\frac{\left(-2+\lambda_{S}^{j}\right)^{2}}{h^{2}\left(10+\lambda_{S}^{j}\right)}$. As a result, $\mathbf{A}-\mathbf{A H}$ is a symmetric and positive definite matrix.

Further, for $\mathbf{u} \in \mathcal{W}_{h}^{0}$, we get $\mathbf{u}^{T}(\mathbf{A}-\mathbf{A H}) \mathbf{u} \geq 0$, which completes the proof.

Lemma 4.4 (cf. [24]) For any mesh function $\mathbf{u}^{n} \in \mathcal{W}_{h}^{0}$, there is

$$
\left\|\mathbf{u}^{n+1}\right\|^{2}-\left\|\mathbf{u}^{n}\right\|^{2} \leq \tau\left[\left\|\delta_{t} \mathbf{u}^{n}\right\|^{2}+\frac{1}{2}\left(\left\|\mathbf{u}^{n}\right\|^{2}+\left\|\mathbf{u}^{n+1}\right\|^{2}\right)\right]
$$

Lemma 4.5 (Discrete Sobolev's inequality [34]) Suppose that $\left\{u_{j}\right\}$ is mesh functions. Given $\epsilon>0$, there exists a constant $C$ dependent on $\epsilon$ such that

$$
\|\mathbf{u}\|_{\infty} \leq \epsilon\left\|\delta_{x} \mathbf{u}\right\|+C\|\mathbf{u}\|
$$

Lemma 4.6 Suppose that $\phi_{0} \in H_{0}^{1}, \phi_{1} \in L_{2}, \beta(x) \geq 0, F(s) \geq 0, s \in[0,+\infty), \beta, f^{\prime} \in C^{1}(\mathbb{R})$, and $\frac{\tau^{2}}{h^{2}}<\frac{1}{3}$. Then the following estimates hold:

$$
\left\|\mathbf{u}^{n}\right\| \leq C, \quad\left\|\delta_{x} \mathbf{u}^{n}\right\| \leq C, \quad\left\|\mathbf{u}^{n}\right\|_{\infty} \leq C .
$$


Proof It follows from Theorem 3.3 that there exists a constant $C$ such that

$$
\begin{aligned}
E^{n}= & \left\|\delta_{t} \mathbf{u}^{n}\right\|^{2}+\frac{1}{2}\left(\left\|\mathbf{R} \mathbf{u}^{n}\right\|^{2}+\left\|\mathbf{R} \mathbf{u}^{n+1}\right\|^{2}\right)-\frac{\tau^{2}}{2}\left\|\mathbf{R} \delta_{t} \mathbf{u}^{n}\right\|^{2} \\
& +\frac{h}{2} \sum_{j=1}^{J-1} \beta_{j}\left(F\left(\left|u_{j}^{n}\right|^{2}\right)+F\left(\left|u_{j}^{n+1}\right|^{2}\right)\right)=C
\end{aligned}
$$

Applying Lemma 3.1 and Lemma 4.2, we can deduce that

$$
-\frac{\tau^{2}}{2}\left\|\mathbf{R} \delta_{t} \mathbf{u}^{n}\right\|^{2} \geq-\frac{3 \tau^{2}}{h^{2}}\left\|\delta_{t} \mathbf{u}^{n}\right\|^{2}
$$

Substituting (4.5) into (4.4), we obtain that

$$
\left\|\delta_{t} \mathbf{u}^{n}\right\|^{2}+\frac{1}{2}\left(\left\|\mathbf{R} \mathbf{u}^{n+1}\right\|^{2}+\left\|\mathbf{R} \mathbf{u}^{n}\right\|^{2}\right)-\frac{3 \tau^{2}}{h^{2}}\left\|\delta_{t} \mathbf{u}^{n}\right\|^{2} \leq C .
$$

Furthermore, inequality (4.6) can be rewritten as

$$
\left(1-\frac{3 \tau^{2}}{h^{2}}\right)\left\|\delta_{t} \mathbf{u}^{n}\right\|^{2}+\frac{1}{2}\left(\left\|\mathbf{R} \mathbf{u}^{n+1}\right\|^{2}+\left\|\mathbf{R} \mathbf{u}^{n}\right\|^{2}\right) \leq C .
$$

Noting that $\left(1-\frac{3 \tau^{2}}{h^{2}}\right)>0$, we have

$$
\left\|\delta_{t} \mathbf{u}^{n}\right\| \leq C, \quad\left\|\mathbf{R} \mathbf{u}^{n}\right\| \leq C
$$

Applying Lemma 4.4 to $\left\|\delta_{t} \mathbf{u}^{n}\right\| \leq C$, we obtain $\left\|\mathbf{u}^{n}\right\| \leq C$. Using Lemma 4.3, we have $\left\|\delta_{x} \mathbf{u}^{n}\right\| \leq C$. Moreover, by Lemma 4.5 , it holds that

$$
\left\|\mathbf{u}^{n}\right\|_{\infty} \leq C
$$

Therefore, the proof is completed.

\section{Convergence and stability of the difference scheme}

In this section, we focus on the convergence and stability of the numerical scheme. Firstly, we define the truncation error $\mathrm{Er}_{j}^{n}$ as

$$
\begin{aligned}
E r_{j}^{n}= & \delta_{t}^{2} U_{j}^{n}-A_{h}^{-1} \delta_{x}^{2} U_{j}^{n}+\mathrm{i} \alpha \delta_{\hat{t}} U_{j}^{n} \\
& +\left[\beta\left(x_{j}\right) \frac{F\left(\left|U_{j}^{n+1}\right|^{2}\right)-F\left(\left|U_{j}^{n-1}\right|^{2}\right)}{\left|U_{j}^{n+1}\right|^{2}-\left|U_{j}^{n-1}\right|^{2}} \frac{U_{j}^{n+1}+U_{j}^{n-1}}{2}\right] .
\end{aligned}
$$

By Taylor's expansion, it is easy to check that

$$
\left|E r_{j}^{n}\right| \leq C\left(\tau^{2}+h^{4}\right)
$$

Before the proof of convergence, we introduce discrete Gronwall's inequality. 
Lemma 5.1 (Discrete Gronwall's inequality [35]) Suppose that the discrete function $w_{n}$ satisfies the recurrence formula

$$
w_{n}-w_{n-1} \leq a \tau w_{n}+b \tau w_{n-1}+c_{n} \tau,
$$

where $a, b$ and $c_{n}(n=1, \ldots, N)$ are nonnegative constants. Then

$$
\max _{1 \leq n \leq N}\left|w_{n}\right| \leq\left(w_{0}+\tau \sum_{k=1}^{N} c_{k}\right) e^{2(a+b) T},
$$

where $\tau$ is small such that $(a+b) \tau \leq \frac{N-1}{2 N}(N>1)$.

Theorem 5.2 Suppose that $\phi_{0} \in H_{0}^{1}, \phi_{1} \in L_{2}, \beta(x) \geq 0, F(s) \geq 0, s \in[0,+\infty), \beta, f^{\prime} \in C^{1}(\mathbb{R})$, and $\frac{\tau^{2}}{h^{2}}<\frac{1}{3}$, then the solution $u^{n}$ of difference problem (2.7)-(2.9) converges to the solution of problem (1.1)-(1.3) with order $\mathcal{O}\left(\tau^{2}+h^{4}\right)$ in the maximal norm.

Proof Let $\mathbf{E r}^{n}=\left(E r_{1}^{n}, \ldots, E r_{J-1}^{n}\right)^{T}$ and $\mathbf{e}^{n}=\mathbf{U}^{n}-\mathbf{u}^{n}$. Firstly, subtracting (2.2) from the vector form of (5.1), the error equations satisfy

$$
\begin{aligned}
\mathbf{E r}^{n}= & \delta_{t}^{2} \mathbf{e}^{n}-\mathbf{H A} \mathbf{e}^{n}+\mathrm{i} \alpha \delta_{\hat{t}} \mathbf{e}^{n}+G\left(\mathbf{U}^{n}\right) \frac{\mathbf{U}^{n+1}+\mathbf{U}^{n-1}}{2}-G\left(\mathbf{u}^{n}\right) \frac{\mathbf{u}^{n+1}+\mathbf{u}^{n-1}}{2} \\
= & \delta_{t}^{2} \mathbf{e}^{n}-\mathbf{H A} \mathbf{e}^{n}+\mathrm{i} \alpha \delta_{\hat{t}} \mathbf{e}^{n}+G\left(\mathbf{U}^{n}\right) \frac{\mathbf{e}^{n+1}+\mathbf{e}^{n-1}}{2} \\
& +\left(G\left(\mathbf{U}^{n}\right)-G\left(\mathbf{u}^{n}\right)\right) \frac{\mathbf{u}^{n+1}+\mathbf{u}^{n-1}}{2} .
\end{aligned}
$$

Computing the inner product with both sides of (5.2) with $\delta_{\hat{t}} \mathbf{e}^{n}$ and considering the real part, we obtain

$$
\begin{aligned}
\operatorname{Re}\left(\mathbf{E r}^{n}, \delta_{t} \mathbf{e}^{n}\right) & \\
= & \frac{1}{2 \tau}\left(\left\|\delta_{t} \mathbf{e}^{n}\right\|^{2}-\left\|\delta_{t} \mathbf{e}^{n-1}\right\|^{2}\right)+\frac{1}{4 \tau}\left(\left\|\mathbf{R e}^{n+1}\right\|^{2}-\left\|\mathbf{R} \mathbf{e}^{n-1}\right\|^{2}\right) \\
& -\frac{\tau}{4}\left(\left\|\mathbf{R} \delta_{t} \mathbf{e}^{n}\right\|^{2}-\left\|\mathbf{R} \delta_{t} \mathbf{e}^{n-1}\right\|^{2}\right)+\operatorname{Re}\left(G\left(\mathbf{U}^{n}\right) \frac{\mathbf{e}^{n+1}+\mathbf{e}^{n-1}}{2}, \delta_{\hat{t}} \mathbf{e}^{n}\right) \\
& +\operatorname{Re}\left(\left(G\left(\mathbf{U}^{n}\right)-G\left(\mathbf{u}^{n}\right)\right) \frac{\mathbf{u}^{n+1}+\mathbf{u}^{n-1}}{2}, \delta_{\hat{t}} \mathbf{e}^{n}\right) .
\end{aligned}
$$

Noticing that $\left|G\left(\mathbf{U}^{n}\right)\right| \leq C$ and $f^{\prime}(s) \in C^{1}$, we have

$$
\begin{aligned}
& \operatorname{Re}\left(\mathbf{E r}^{n}, \delta_{\hat{t}} \mathbf{e}^{n}\right) \leq C\left(\left\|\mathbf{E r}^{n}\right\|^{2}+\left\|\delta_{t} \mathbf{e}^{n}\right\|^{2}+\left\|\delta_{t} \mathbf{e}^{n-1}\right\|^{2}\right), \\
& \operatorname{Re}\left(G\left(\mathbf{U}^{n}\right) \frac{\mathbf{e}^{n+1}+\mathbf{e}^{n-1}}{2}, \delta_{\hat{t}} \mathbf{e}^{n}\right) \leq C\left(\left\|\mathbf{e}^{n+1}\right\|^{2}+\left\|\mathbf{e}^{n-1}\right\|^{2}+\left\|\delta_{t} \mathbf{e}^{n}\right\|^{2}+\left\|\delta_{t} \mathbf{e}^{n-1}\right\|^{2}\right), \\
& \operatorname{Re}\left(\left(G\left(\mathbf{U}^{n}\right)-G\left(\mathbf{u}^{n}\right)\right) \frac{\mathbf{u}^{n+1}+\mathbf{u}^{n-1}}{2}, \delta_{t} \mathbf{e}^{n}\right) \leq C\left(\left\|\delta_{t} \mathbf{e}^{n}\right\|^{2}+\left\|\delta_{t} \mathbf{e}^{n-1}\right\|^{2}+\left\|\mathbf{e}^{n}\right\|^{2}\right) .
\end{aligned}
$$

It follows from Lemma 4.4 that

$$
\frac{1}{\tau}\left(\left\|\mathbf{e}^{n}\right\|^{2}-\left\|\mathbf{e}^{n-1}\right\|^{2}\right) \leq C\left(\left\|\delta_{t} \mathbf{e}^{n-1}\right\|^{2}+\left\|\mathbf{e}^{n-1}\right\|^{2}+\left\|\mathbf{e}^{n}\right\|^{2}\right) .
$$


Substituting (5.4)-(5.6) into (5.3) and combining with (5.7), we obtain

$$
\begin{aligned}
& \frac{1}{2 \tau}\left(\left\|\delta_{t} \mathbf{e}^{n}\right\|^{2}-\left\|\delta_{t} \mathbf{e}^{n-1}\right\|^{2}\right)+\frac{1}{4 \tau}\left(\left\|\mathbf{R} \mathbf{e}^{n+1}\right\|^{2}-\left\|\mathbf{R} \mathbf{e}^{n-1}\right\|^{2}\right) \\
& \quad+\frac{1}{\tau}\left(\left\|\mathbf{e}^{n}\right\|^{2}-\left\|\mathbf{e}^{n-1}\right\|^{2}\right)-\frac{\tau}{4}\left(\left\|\mathbf{R} \delta_{t} \mathbf{e}^{n}\right\|^{2}-\left\|\mathbf{R} \delta_{t} \mathbf{e}^{n-1}\right\|^{2}\right) \\
& \leq C\left(\left\|\mathbf{E} \mathbf{r}^{n}\right\|^{2}+\left\|\delta_{t} \mathbf{e}^{n}\right\|^{2}+\left\|\delta_{t} \mathbf{e}^{n-1}\right\|^{2}+\left\|\mathbf{e}^{n+1}\right\|^{2}+\left\|\mathbf{e}^{n}\right\|^{2}+\left\|\mathbf{e}^{n-1}\right\|^{2}\right) .
\end{aligned}
$$

Summing inequalities (5.8) up for $n$ leads to

$$
\begin{gathered}
\left\|\delta_{t} \mathbf{e}^{n}\right\|^{2}+\frac{1}{2}\left(\left\|\mathbf{R e}^{n+1}\right\|^{2}+\left\|\mathbf{R e}^{n}\right\|^{2}\right)+2\left\|\mathbf{e}^{n}\right\|^{2}-\frac{\tau^{2}}{2}\left\|\mathbf{R} \delta_{t} \mathbf{e}^{n}\right\|^{2} \\
\leq \tau C \sum_{i=1}^{n}\left[\left\|\delta_{t} \mathbf{e}^{i}\right\|^{2}+\frac{1}{2}\left(\left\|\mathbf{R e}^{i+1}\right\|^{2}+\left\|\mathbf{R} \mathbf{e}^{i}\right\|^{2}\right)+2\left\|\mathbf{e}^{i}\right\|^{2}\right. \\
\left.-\frac{\tau^{2}}{2}\left\|\mathbf{R} \delta_{t} \mathbf{e}^{i}\right\|^{2}\right]+C T\left(\tau^{2}+h^{4}\right)^{2} .
\end{gathered}
$$

According to Lemma 5.1, it yields that

$$
\begin{aligned}
& \left\|\delta_{t} \mathbf{e}^{n}\right\|^{2}+\frac{1}{2}\left(\left\|\mathbf{R e}^{n+1}\right\|^{2}+\left\|\mathbf{R e}^{n}\right\|^{2}\right)+2\left\|\mathbf{e}^{n}\right\|^{2}-\frac{\tau^{2}}{2}\left\|\mathbf{R} \delta_{t} \mathbf{e}^{n}\right\|^{2} \\
& \leq C T\left(\tau^{2}+h^{4}\right)^{2} .
\end{aligned}
$$

Moreover, combining inequality (4.5) and using Lemma 4.2, we get

$$
\begin{aligned}
& \left(1-\frac{3 \tau^{2}}{h^{2}}\right)\left\|\delta_{t} \mathbf{e}^{n}\right\|^{2}+\frac{1}{2}\left(\left\|\mathbf{R e}^{n+1}\right\|^{2}+\left\|\mathbf{R e}^{n}\right\|^{2}\right)+2\left\|\mathbf{e}^{n}\right\|^{2} \\
& \quad \leq C T\left(\tau^{2}+h^{4}\right)^{2} .
\end{aligned}
$$

The rest of the proof of convergence is similar to that of Theorem 4.6. As a result, we have

$$
\left\|\mathbf{e}^{n}\right\|_{\infty} \leq \mathcal{O}\left(\tau^{2}+h^{4}\right)
$$

The proof is completed.

Similarly, we present the stability of difference scheme (2.7)-(2.9).

Theorem 5.3 Under the conditions of Theorem 5.2, the difference scheme (2.7)-(2.9) is stable for the initial data.

\section{Some extensions}

In this section, we present several other conservative compact schemes, which conserve the discrete conservative law. Moreover, we use Richardson extrapolation to improve the accuracy in the temporal direction. 


\subsection{Several conservative compact schemes}

In this subsection, the proofs of the boundedness of numerical solutions, the stability and convergence of numerical schemes are similar to those in the previous sections. We only list the numerical schemes and the discrete energy conservative laws for all schemes.

Scheme 1

$$
\begin{aligned}
& A_{h} \delta_{t}^{2} u_{j}^{n}-\delta_{x}^{2} \frac{u_{j}^{n+1}+u_{j}^{n-1}}{2}+\mathrm{i} \alpha A_{h} \delta_{\hat{t}} u_{j}^{n} \\
& \quad+A_{h}\left[\beta\left(x_{j}\right) \frac{F\left(\left|u_{j}^{n+1}\right|^{2}\right)-F\left(\left|u_{j}^{n-1}\right|^{2}\right)}{\left|u_{j}^{n+1}\right|^{2}-\left|u_{j}^{n-1}\right|^{2}} \frac{u_{j}^{n+1}+u_{j}^{n-1}}{2}\right]=0, \\
& \quad 1 \leq j \leq J-1,1 \leq n \leq N-1, \\
& u_{j}^{0}=\phi_{0}\left(x_{j}\right), \quad \delta_{\hat{t}}\left(x_{j}, 0\right)=\phi_{1}\left(x_{j}\right), \quad 0 \leq j \leq J, \\
& u_{0}^{n}=u_{J}^{n}=0, \quad 0 \leq n \leq N .
\end{aligned}
$$

The discrete conservative law of Scheme 1 is

$$
E^{n}=\left\|\delta_{t} \mathbf{u}^{n}\right\|^{2}+\frac{1}{2}\left(\left\|\mathbf{R} \mathbf{u}^{n}\right\|^{2}+\left\|\mathbf{R} \mathbf{u}^{n+1}\right\|^{2}\right)+\frac{h}{2} \sum_{j=1}^{J-1} \beta_{j}\left[F\left(\left|u_{j}^{n}\right|^{2}\right)+F\left(\left|u_{j}^{n+1}\right|^{2}\right)\right]=E^{0} .
$$

Scheme 2

$$
\begin{aligned}
& A_{h} \delta_{t}^{2} u_{j}^{n}-\delta_{x}^{2} \frac{u_{j}^{n+1}+u_{j}^{n-1}}{2}+\mathrm{i} \alpha A_{h} \delta_{\hat{t}} u_{j}^{n} \\
& \quad+A_{h}\left[\beta\left(x_{j}\right) \frac{F\left(\frac{\left|u_{j}^{n+1}\right|^{2}+\left|u_{j}^{n}\right|^{2}}{2}\right)-F\left(\frac{\left|u_{j}^{n}\right|^{2}+\left|u_{j}^{n-1}\right|^{2}}{2}\right)}{\left|u_{j}^{n+1}\right|^{2}-\left|u_{j}^{n-1}\right|^{2}}\left(u_{j}^{n+1}+u_{j}^{n-1}\right)\right]=0, \\
& \quad 1 \leq j \leq J-1,1 \leq n \leq N-1, \\
& u_{j}^{0}=\phi_{0}\left(x_{j}\right), \quad \delta_{\hat{t}}\left(x_{j}, 0\right)=\phi_{1}\left(x_{j}\right), \quad 0 \leq j \leq J, \\
& u_{0}^{n}=u_{J}^{n}=0, \quad 0 \leq n \leq N .
\end{aligned}
$$

The discrete conservative law of Scheme 2 is

$$
E^{n}=\left\|\delta_{t} \mathbf{u}^{n}\right\|^{2}+\frac{1}{2}\left(\left\|\mathbf{R} \mathbf{u}^{n}\right\|^{2}+\left\|\mathbf{R} \mathbf{u}^{n+1}\right\|^{2}\right)+h \sum_{j=1}^{J-1} \beta_{j} F\left(\frac{\left|u_{j}^{n+1}\right|^{2}+\left|u_{j}^{n}\right|^{2}}{2}\right)=E^{0} .
$$

\section{Scheme 3}

$$
\begin{aligned}
& A_{h} \delta_{t}^{2} u_{j}^{n}-\delta_{x}^{2} u_{j}^{n}+\mathrm{i} \alpha A_{h} \delta_{\hat{t}} u_{j}^{n} \\
& \quad+A_{h}\left[\beta\left(x_{j}\right) \frac{F\left(\frac{\left|u_{j}^{n+1}\right|^{2}+\left|u_{j}^{n}\right|^{2}}{2}\right)-F\left(\frac{\left|u_{j}^{n}\right|^{2}+\left|u_{j}^{n-1}\right|^{2}}{2}\right)}{\left|u_{j}^{n+1}\right|^{2}-\left|u_{j}^{n-1}\right|^{2}}\left(u_{j}^{n+1}+u_{j}^{n-1}\right)\right]=0, \\
& \quad 1 \leq j \leq J-1,1 \leq n \leq N-1, \\
& u_{j}^{0}=\phi_{0}\left(x_{j}\right), \quad \delta_{\hat{t}}\left(x_{j}, 0\right)=\phi_{1}\left(x_{j}\right), \quad 0 \leq j \leq J, \\
& u_{0}^{n}=u_{J}^{n}=0, \quad 0 \leq n \leq N .
\end{aligned}
$$


The discrete conservative law of Scheme 3 is

$$
\begin{aligned}
E^{n}= & \left\|\delta_{t} \mathbf{u}^{n}\right\|^{2}+\frac{1}{2}\left(\left\|\mathbf{R} \mathbf{u}^{n}\right\|^{2}+\left\|\mathbf{R} \mathbf{u}^{n+1}\right\|^{2}\right)-\frac{\tau^{2}}{2}\left\|\mathbf{R} \delta_{t} \mathbf{u}^{n}\right\|^{2} \\
& +h \sum_{j=1}^{J-1} \beta_{j} F\left(\frac{\left|u_{j}^{n+1}\right|^{2}+\left|u_{j}^{n}\right|^{2}}{2}\right)=E^{0} .
\end{aligned}
$$

Scheme 4

$$
\begin{aligned}
& A_{h} \delta_{t}^{2} \frac{u_{j}^{n+1}+u_{j}^{n}}{2}-\delta_{x}^{2} \frac{u_{j}^{n+1}+u_{j}^{n}}{2}+\mathrm{i} \alpha A_{h} \delta_{t} u_{j}^{n} \\
& \quad+A_{h}\left[\beta\left(x_{j}\right) \frac{F\left(\left|u_{j}^{n+1}\right|^{2}\right)-F\left(\left|u_{j}^{n}\right|^{2}\right)}{\left|u_{j}^{n+1}\right|^{2}-\left|u_{j}^{n}\right|^{2}} \frac{u_{j}^{n+1}+u_{j}^{n}}{2}\right]=0, \\
& \quad 1 \leq j \leq J-1,1 \leq n \leq N-1, \\
& u_{j}^{0}=\phi_{0}\left(x_{j}\right), \quad \delta_{\hat{t}}\left(x_{j}, 0\right)=\phi_{1}\left(x_{j}\right), \quad 0 \leq j \leq J, \\
& u_{0}^{n}=u_{J}^{n}=0, \quad 0 \leq n \leq N .
\end{aligned}
$$

The discrete conservative law of Scheme 4 is

$$
\begin{aligned}
E^{n}= & \frac{1}{2}\left(\left\|\delta_{t} \mathbf{u}^{n}\right\|^{2}+\left\|\delta_{\bar{t}} \mathbf{u}^{n}\right\|^{2}\right)-\frac{\tau^{2}}{2}\left\|\delta_{t}^{2} \mathbf{u}^{n}\right\|^{2} \\
& +\left\|\mathbf{R} \mathbf{u}^{n}\right\|^{2}+h \sum_{j=1}^{J-1} \beta_{j} F\left(\left|u_{j}^{n}\right|^{2}\right)=E^{0} .
\end{aligned}
$$

\subsection{Richardson extrapolation}

In order to improve the accuracy in the temporal direction, we apply Richardson extrapolation, which is given by a linear combination of numerical solutions under different mesh grids. Applying Taylor's expansion, we obtain that the main term of truncation error $\operatorname{Er}_{j}^{n}$ is $\mathcal{O}\left(\tau^{2}+\tau^{4}+h^{4}\right)$. Hence, we use the following Richardson method (see [36]):

$$
\left(u_{R}\right)_{j}^{n}=\frac{4}{3} u_{j}^{2 n}\left(h, \frac{\tau}{2}\right)-\frac{1}{3} u_{j}^{n}(h, \tau),
$$

where $u_{j}^{n}(h, \tau)$ is the numerical solutions at the grid point $\left(x_{j}, t_{n}\right)$ with spatial step size $h$ and temporal step size $\tau$, and $u_{j}^{2 n}\left(h, \frac{\tau}{2}\right)$ is the numerical solutions at the grid point $\left(x_{j}, t_{n}\right)$ with spatial step size $h$ and temporal step size $\frac{\tau}{2}$. Here, the convergence order of the Richardson method is $\mathcal{O}\left(\tau^{4}+h^{4}\right)$.

\section{Numerical experiments}

In this section, we use serval numerical experiments to confirm the discrete conservation law, convergence as well as stability. Due to the implicitness and nonlinearity in scheme (2.7)-(2.9), the split iterative algorithm [37] is used to resolve this problem. We take $10^{-8}$ as the iterative tolerance. 
Example 1 We present some accuracy tests by considering the following equation:

$$
\begin{aligned}
& u_{t t}-u_{x x}+\mathrm{i} u_{t}+|u|^{4} u=f(x, t), \quad(x, t) \in(0,1) \times(0,1), \\
& u(x, 0)=x(x-1), \quad u_{t}(x, 0)=-\mathrm{i} x(x-1), \quad x \in[0,1], \\
& u(0, t)=u(1, t)=0, \quad t \in[0,1],
\end{aligned}
$$

where $f(x, t)=-2 e^{-\mathrm{i} t}+x^{5}(x-1)^{5} e^{-\mathrm{i} t}$.

The exact solution of the problem is

$$
u(x, t)=e^{-\mathrm{i} t}(x-1) x
$$

In this example, the maximum norm is defined as follows:

$$
\begin{aligned}
& \|\operatorname{err} 1\|_{\infty}=\max _{\substack{0 \leq j \leq J \\
0 \leq n \leq N}}\left|u\left(x_{j}, t_{n}\right)-u_{j}^{n}\right| \\
& \left\|\operatorname{err}_{R} 1\right\|_{\infty}=\max _{\substack{0 \leq j \leq J \\
0 \leq n \leq N}}\left|u\left(x_{j}, t_{n}\right)-\left(\frac{4}{3} u_{j}^{2 n}\left(h, \frac{\tau}{2}\right)-\frac{1}{3} u_{j}^{n}(h, \tau)\right)\right| .
\end{aligned}
$$

Scheme (2.7)-(2.9) with $\tau=h^{2}$ is applied to solve (7.1)-(7.3). The numerical errors are plotted in Fig. 1. It indicates that the convergence order of the scheme is $\mathcal{O}\left(\tau^{2}+h^{4}\right)$. To improve temporal accuracy, Richardson extrapolation with $\tau=h$ is applied to solve the problem. The numerical errors are given in Fig. 2. Clearly, it implies that the convergence

Figure 1 The convergence order of scheme (2.7)-(2.9) for Example 1

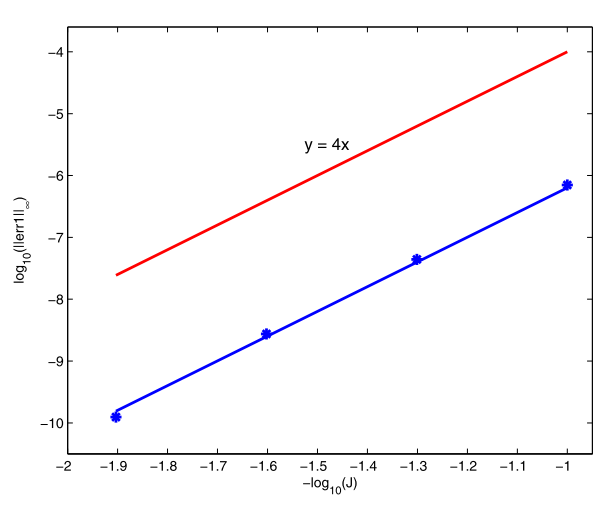

Figure 2 The convergence order of Richardson extrapolation for Example 1

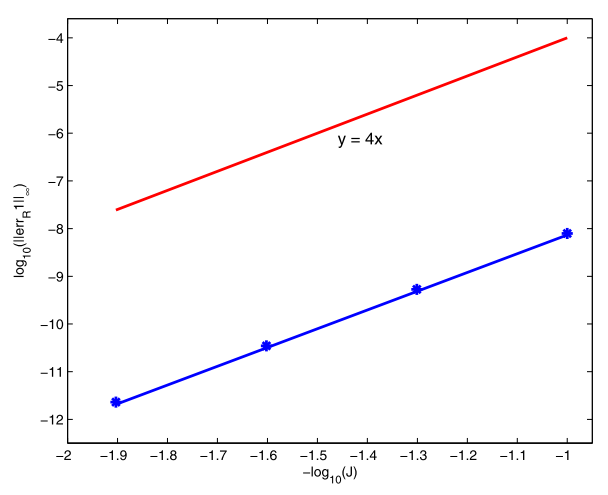


Figure 3 The long-term behavior of numerical solutions corresponding to scheme (2.7)-(2.9) from $t=0$ to $t=10$ at $h=0.01, \tau=h^{2}$ for Example 1

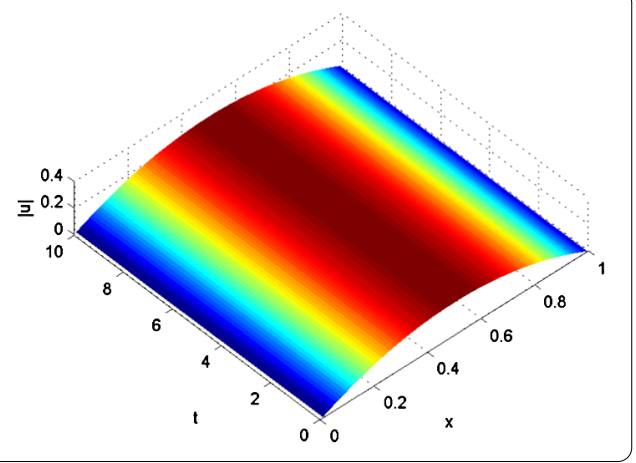

Figure 4 The long-term behavior of numerical solutions corresponding to Richardson extrapolation from $t=0$ to $t=10$ at $h=0.01, \tau=h^{2}$ for Example 1

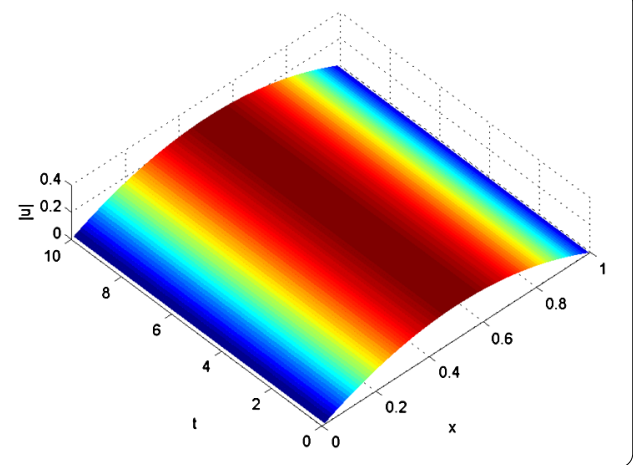

Figure 5 The movement of $|u|$ of scheme (2.7)-(2.9) at $t=2,5,10$ for Example 1

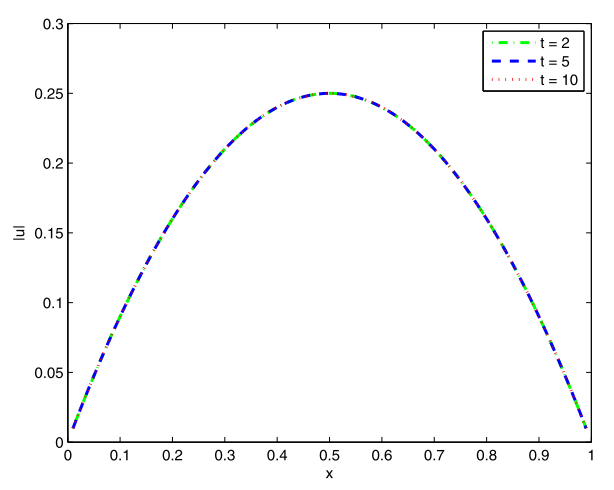

order of the method is $\mathcal{O}\left(\tau^{4}+h^{4}\right)$. We also numerically solve the problem with $h=0.01$, $\tau=h^{2}$, and $T=10$. Figs. 3 and 4 indicate the long-term behavior of numerical solutions with respect to scheme (2.7)-(2.9) and Richardson extrapolation, respectively. Figs. 5 and 6 further show the movement of $|u|$ at different times, i.e., $t=2,5,10$. These figures further imply that the numerical schemes are effective.

Example 2 In order to further confirm the theoretical results, we present the following tests by considering the following equation:

$$
\begin{aligned}
& u_{t t}-u_{x x}+\mathrm{i} u_{t}+|u|^{2} u=0, \quad(x, t) \in(-40,40) \times(0,1), \\
& u(x, 0)=(1+\mathrm{i}) x e^{-10(1-x)^{2}}, \quad u_{t}(x, 0)=0, \quad x \in[-40,40] .
\end{aligned}
$$


Figure 6 The movement of $|u|$ of Richardson extrapolation at $t=2,5,10$ for Example 1

Figure 7 The convergence order of scheme (2.7)-(2.9) for Example 2
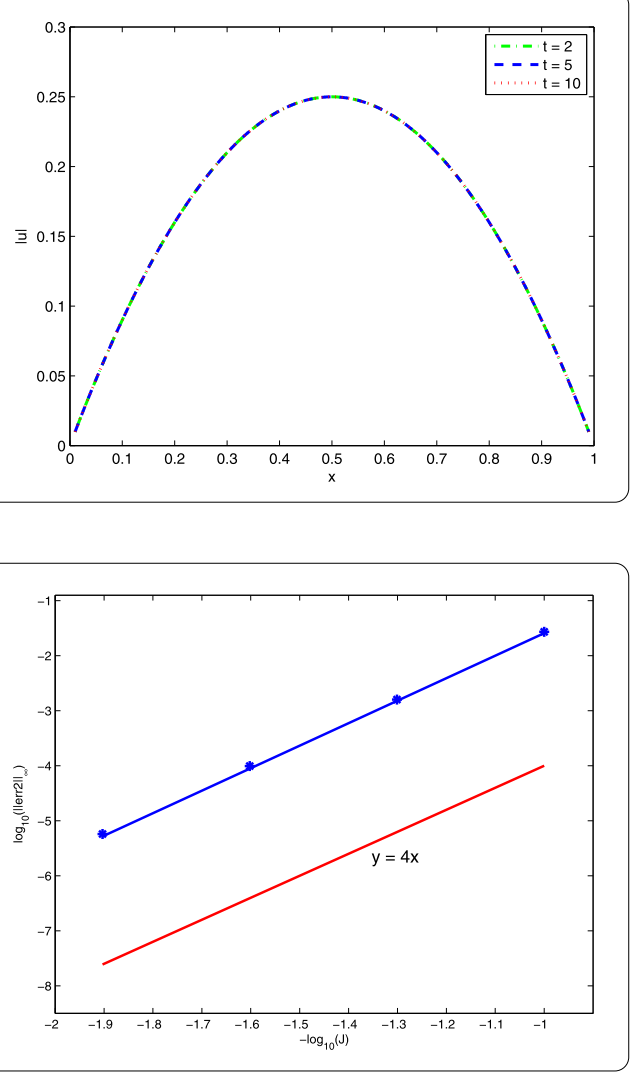

Figure 8 The convergence order of Richardson extrapolation for Example 2

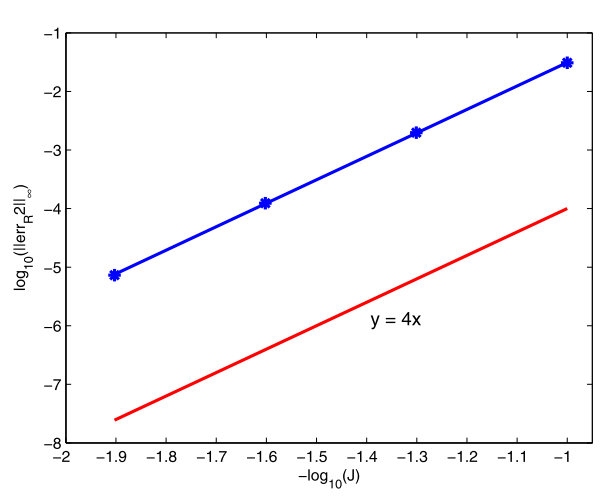

The maximum norm in this test is defined as follows:

$$
\begin{aligned}
& \|\operatorname{err} 2\|_{\infty}=\max _{\substack{0 \leq j \leq J \\
0 \leq n \leq N}}\left|u\left(x_{j}, t_{n}\right)-u_{j}^{n}\right|, \\
& \left\|\operatorname{err}_{R} 2\right\|_{\infty}=\max _{\substack{0 \leq j \leq J \\
0 \leq n \leq N}}\left|u\left(x_{j}, t_{n}\right)-\left(\frac{4}{3} u_{j}^{2 n}\left(h, \frac{\tau}{2}\right)-\frac{1}{3} u_{j}^{n}(h, \tau)\right)\right| .
\end{aligned}
$$

Since the exact solution of the problem is unknown, we take the numerical solution with $h=0.0125, \tau=h^{2}$ as the reference solution. To numerically solve problem, we still apply scheme (2.7)-(2.9) with $\tau=h^{2}$ and Richardson extrapolation with $\tau=h$. The numerical errors with different methods are shown in Figs. 7 and 8. Again, the numerical 
Figure 9 The wave propagation of scheme (2.7)-(2.9) at $h=0.1, \tau=h^{2}$ for Example 2

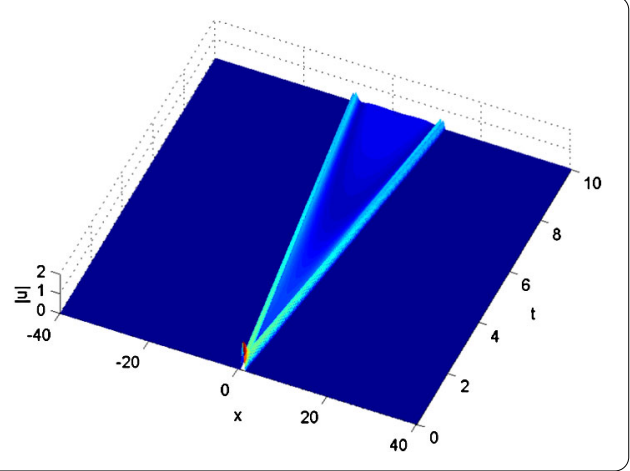

Figure 10 The wave propagation of Richardson extrapolation at $h=0.1, \tau=h^{2}$ for Example 2

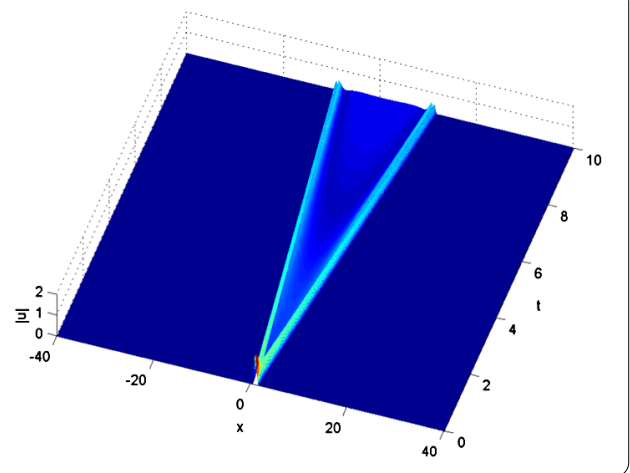

Figure 11 The movement of $|u|$ of scheme (2.7)-(2.9) at $t=2,5,10$ for Example 2

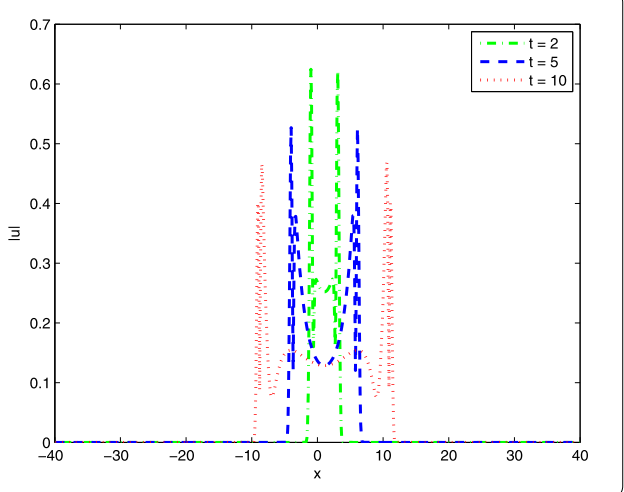

results indicate that the convergence order of scheme (2.7)-(2.9) is $\mathcal{O}\left(\tau^{2}+h^{4}\right)$ and the convergence order of the scheme with Richardson extrapolation is $\mathcal{O}\left(\tau^{4}+h^{4}\right)$. Moreover, Figs. 9 and 10 display the wave propagation of scheme (2.7)-(2.9) and Richardson extrapolation with $h=0.1, \tau=h^{2}$, and $T=10$, respectively. Figs. 11 and 12 show the movement of $|u|$ of scheme (2.7)-(2.9) and Richardson extrapolation at $t=2,5,10$, respectively. In order to further confirm the discrete conservation law, we choose $h=0.05, \tau=h^{2}$ to compute the numerical solution from $t=0$ to $t=10$. The discrete energy is listed in Table 1 . Clearly, it confirms the energy-conserving property of the fully discrete numerical scheme. 
Figure 12 The movement of $|u|$ of Richardson extrapolation at $t=2,5,10$ for Example 2

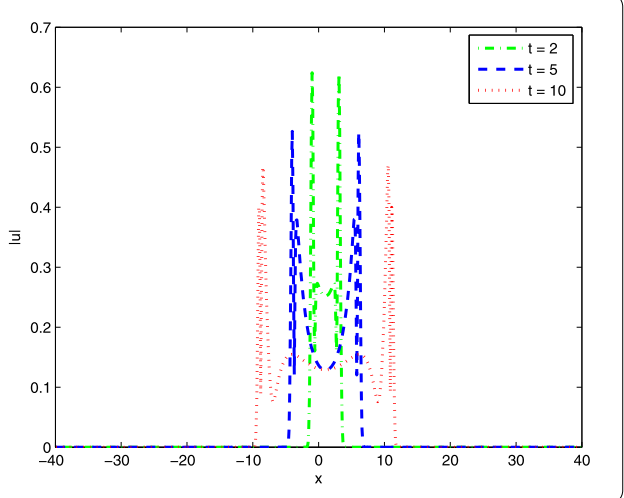

Table 1 The discrete energy $E$ at different time with $h=0.05, \tau=h^{2}$ for Example 2

\begin{tabular}{llrl}
\hline$t$ & $E$ & $t$ & $E$ \\
\hline 1 & 9.123106257391363 & 6 & 9.123106257330761 \\
2 & 9.123106257374163 & 7 & 9.123106257328724 \\
3 & 9.123106257358231 & 8 & 9.123106257329276 \\
4 & 9.123106257345416 & 9 & 9.123106257332394 \\
5 & 9.123106257336049 & 10 & 9.123106257338352 \\
\hline
\end{tabular}

\section{Conclusion}

In this work, we presented several conservative compact schemes for solving a class of nonlinear Schrödinger equations with wave operator. By the energy method, it was proved that the numerical solution is bounded and numerical scheme (2.7)-(2.9) is convergent and stable. The convergence rate is $\mathcal{O}\left(\tau^{2}+h^{4}\right)$ in $l_{\infty}$ norm. Furthermore, the order of scheme (2.7)-(2.9) is improved to $\mathcal{O}\left(\tau^{4}+h^{4}\right)$ by applying Richardson extrapolation. Finally, all the numerical results show that difference scheme (2.7)-(2.9) and Richardson extrapolation are efficient.

\section{Acknowledgements}

We would like to thank Prof. Jinqiao Duan and Dr. Xiaoli Chen for helpful discussions.

\section{Funding}

This work was partly supported by the National Science Foundation (grant No. 1620449) and the National Natural Science Foundation of China (grant Nos. 11771162, 11531006, and 11771449).

\section{Availability of data and materials}

Not applicable.

\section{Competing interests}

All authors declare that none of them has competing interests.

\section{Authors' contributions}

XC designed the numerical schemes and wrote the first draft of the manuscript. FW considered the numerical simulations. Both authors read and approved the final version of the manuscript.

\section{Publisher's Note}

Springer Nature remains neutral with regard to jurisdictional claims in published maps and institutional affiliations.

Received: 4 December 2017 Accepted: 12 March 2018 Published online: 27 March 2018

\section{References}

1. Machihara, S., Nakanishi, K., Ozawa, T.: Nonrelativistic limit in the energy space for nonlinear Klein-Gordon equations. Math. Ann. 322, 603-621 (2002) 
2. Schoene, A.Y.: On the nonrelativistic limits of the Klein-Gordon and Dirac equations. J. Math. Anal. Appl. 71, 36-47 (1979)

3. Bergé, L., Colin, T.: A singular perturbation problem for an envelope equation in plasma physics. Physica D 84 437-459 (1995)

4. Liao, L., Ji, G., Tang, Z., Zhang, H.: Spike-layer simulation for steady-state coupled Schrödinger equations. East Asian J. Appl. Math. 7, 566-582 (2017)

5. Saanouni, T:: Global well-posedness of some high-order focusing semilinear evolution equations with exponential nonlinearity. Adv. Nonlinear Anal. 7, 67-84 (2017)

6. Xin, J: Modeling light bullets with the two-dimensional sine-Gordon equation. Physica D 135, 345-368 (2000)

7. Guo, B., Hua, H.: On the problem of numerical calculation for a class of the system of nonlinear Schrödinger equation with wave operator. J. Numer. Methods Comput. Appl. 4, 258-263 (1983)

8. Holzleitner, M., Kostenko, A., Teschl, G.: Dispersion estimates for spherical Schrödinger equations: the effect of boundary conditions. Opusc. Math. 36(6), 769-786 (2016)

9. Bao, W., Cai, Y.: Uniform error estimates of finite difference methods for the nonlinear Schrödinger equation with wave operator. SIAM J. Numer. Anal. 50, 492-521 (2012)

10. Sun, W., Wang, J.: Optimal error analysis of Crank-Nicolson schemes for a coupled nonlinear Schrödinger system in 3D. J. Comput. Appl. Math. 317, 685-699 (2017)

11. Chang, Q., Jia, E., Sun, W.: Difference schemes for solving the generalized nonlinear Schrödinger equation. J. Comput. Phys. 148, 397-415 (1999)

12. Goubet, O., Hamraoui, E.: Blow-up of solutions to cubic nonlinear Schrödinger equations with defect: the radial case. Adv. Nonlinear Anal. 6(2), 183-197 (2017)

13. Li, D., Wang, J.: Unconditionally optimal error analysis of Crank-Nicolson Galerkin FEMs for a strongly nonlinear parabolic system. J. Sci. Comput. 72, 892-915 (2017)

14. Li, D., Zhang, C., Ran, M.: A linear finite difference scheme for generalized time fractional Burgers equation. Appl. Math. Model. 40, 6069-6081 (2016)

15. Li, D., Liao, H., Sun, W., Wang, J., Zhang, J.: Analysis of L1-Galerkin FEMs for time-fractional nonlinear parabolic problems. Commun. Comput. Phys. 24, 86-103 (2018)

16. Jannelli, A., Ruggieri, M., Speciale, M.P.: Exact and numerical solutions of time-fractional advection-diffusion equation with a nonlinear source term by means of the Lie symmetries. Nonlinear Dyn. (2018). https://doi.org/10.1007/s11071-018-4074-8

17. Zhang, Q., Zhang, C., Wang, L.: The compact and Crank-Nicolson ADI schemes for two-dimensional semilinear multidelay parabolic equations. J. Comput. Appl. Math. 306, 217-230 (2016)

18. Zhang, Q., Mei, M., Zhang, C.: Higher-order linearized multistep finite difference methods for non-Fickian delay reaction-diffusion equations. Int. J. Numer. Anal. Model. 14, 1-19 (2017)

19. Li, D., Wang, J., Zhang, J: Unconditionally convergent L1-Galerkin FEMs for nonlinear time-fractional Schrödinger equations. SIAM J. Sci. Comput. 39, A3067-A3088 (2017)

20. Li, D., Zhang, J., Zhang, Z.: Unconditionally optimal error estimates of a linearized Galerkin method for nonlinear time fractional reaction-subdiffusion equations. J. Sci. Comput. (2018). https://doi.org/10.1007/s10915-018-0642-9

21. Kumar, S., Kumar, D., Singh, J.: Fractional modelling arising in unidirectional propagation of long waves in dispersive media. Adv. Nonlinear Anal. 5(4), 383-394 (2016)

22. Zhang, F., Peréz-Ggarcía, V.M., Vázquez, L.: Numerical simulation of nonlinear Schrödinger equation system: a new conservative scheme. Appl. Math. Comput. 71, 165-177 (1995)

23. Brugnano, L., Zhang, C., Li, D.: A class of energy-conserving Hamiltonian boundary value methods for nonlinear Schrödinger equation with wave operator. Commun. Nonlinear Sci. Numer. Simul. 60, 33-49 (2018)

24. Zhang, L., Chang, Q: A conservative numerical scheme for a class of nonlinear Schrödinger equation with wave operator. Appl. Math. Comput. 145, 602-613 (2003)

25. Wang, T., Zhang, L.: Analysis of some new conservative schemes for nonlinear Schrödinger equation with wave operator. Appl. Math. Comput. 182, 1780-1794 (2006)

26. $\mathrm{Hu}, \mathrm{H} ., \mathrm{Chan}, \mathrm{Y}$ : A conservative difference scheme for two-dimensional nonlinear Schrödinger equation with wave operator. Numer. Methods Partial Differ. Equ. 32, 862-876 (2016)

27. Guo, L., Xu, Y.: Energy conserving local discontinuous Galerkin methods for nonlinear Schrödinger equation with wave operator. J. Sci. Comput. 65, 622-647 (2015)

28. Cao, W., Li, D., Zhang, Z:: Optimal superconvergence of energy conserving local discontinuous Galerkin methods for wave equations. Commun. Comput. Phys. 21, 211-236 (2017)

29. Li, X., Zhang, L., Wang, S.: A compact finite difference scheme for the nonlinear Schrödinger equation with wave operator. Appl. Math. Comput. 219, 3187-3197 (2012)

30. Li, X., Zhang, L., Zhang, T.: A new numerical scheme for the nonlinear Schrödinger equation with wave operator. Appl. Math. Comput. 54, 109-125 (2017)

31. Li, D., Zhang, C., Wen, J.: A note on compact finite difference method for reaction-diffusion equations with delay. Appl. Math. Model. 39, 1749-1754 (2015)

32. Sun, Z: Numerical Methods of the Partial Differential Equations. Science Press, Beijing (2005)

33. Horn, R.A., Johnson, C.R.: Matrix Analysis. Cambridge University Press, Cambridge (2012)

34. Chan, T., Shen, L.: Stability analysis of difference schemes for variable coefficient Schrödinger type equations. SIAM J. Numer. Anal. 24, 336-349 (1981)

35. Zhou, Y: Application of Discrete Functional Analysis to the Finite Difference Methods. International Academic Publishers, Beijing (1990)

36. Deng, D., Zhang, C.: Analysis and application of a compact multistep ADI solver for a class of nonlinear viscous wave equations. Appl. Math. Model. 39, 1033-1049 (2015)

37. Li, D., Zhang, C.: Split Newton iterative algorithm and its application. Appl. Math. Comput. 217, 2260-2265 (2010) 